\title{
Acupuncture Practices and Brain Waves
}

\author{
Mehmet Tuğrul Cabığlu \\ Department of Physiology, Faculty of Medicine, Başkent University, Ankara, Turkey \\ Email: tugcab@gmail.com
}

Received 1 June 2016; accepted 17 June 2016; published 21 June 2016

Copyright (C) 2016 by author and OALib.

This work is licensed under the Creative Commons Attribution International License (CC BY). http://creativecommons.org/licenses/by/4.0/

(c) (i) Open Access

\begin{abstract}
Acupuncture is the most frequently used treatment method among the complementary medicine practices. Acupuncture practices mainly affect the somatic and autonomic nervous systems in the nervous system. It is important to understand the mechanism of action of acupuncture practices especially in the central nervous system. Electroencephalography (EEG) is one of the best techniques to explain the effects of acupuncture on the central nervous system. In the present review, the changes in the EEG by the application of acupuncture to different acupoints were evaluated. It was observed that the application of acupuncture to different acupoints caused different brain activities in different regions of the brain. In addition to this, an increase in the alpha-1 wave frequency due to the stimulation of Baihui (GV 20), Neiguan (P6), and Hegu (LI 4) acupoints in different meridian and regions has indicated that these points have mood modifying and relaxation effects and they can be used in the psychological state changes.
\end{abstract}

\section{Keywords}

Acupuncture, Electroencephalography, Brain Activity, Alfa Wave, Relaxation

Subject Areas: Neuroscience

\section{Introduction}

Among the complementary medicine practices, which are widely used in recent years worldwide, acupuncture is one of the most popular and frequently used practices. Acupuncture has been applied in many disorders, such as psychological condition changes and immune system- and metabolism-related diseases, especially diseases with pain, either alone or as a support to other treatment methods.

\section{Effect's Mechanism of Acupuncture}

When acupuncture needle is inserted into an acupoint, body responds to this situation by activating particularly the nervous system and different systems as well [1] [2]. In the process of "Repair It" command for the tissue damage due to microtrauma occurred by the insertion of acupuncture needle into the tissue, activation of "Pain 
Control" system as a result of increases in the levels of endogenous opioids, serotonin, and noradrenaline in the central nervous system and plasma is the most important mechanism of action of acupuncture [3]-[6]. There are many studies regarding the regulatory effect of acupuncture practices on autonomic nervous system [5] [7] [8].

\section{Brain Waves and EEG}

Electrical recordings obtained from outer surface of the head show a continuous electrical activity. These electrical signals are recorded by electroencephalography (EEG) as brain waves. EEG signals are defined as multidimensional, time domain biological signals that externalize information regarding processes in the brain, especially at the excitatory pyramidal neuron level. Complex cortical generators continuously process a wide range of externally and internally generated stimuli. Furthermore, EEG signals are subject to noise and artefacts. Extracting information from EEG is therefore extremely challenging [9]. Both intensity and the pattern of this electrical activity are determined by the level of excitation of different parts of the brain that could cause sleep or brain disorders such as epilepsy or psychological disorders such as anxiety or depression. The character of the wave depends on the degree of activity in respective parts of the cerebral cortex, and the waves change markedly between the states of wakefulness and sleep and coma [10].

Waves in the EEG can be classified as alpha, beta, theta, and delta waves. Alpha waves are that occur frequencies between 8 and 13 cycles/sec and are found in the EEG of almost all healthy adults when they are awake and not concentrating anything in a resting position. Beta waves occur at frequencies between 14 and 80 cycles/sec in the active state and when the person awake. Theta waves have frequencies between 4 and 7 cycles/ sec. They occur during emotional stress in some adults, particularly during disappointment and frustrations. Theta waves also occur in many brain disorders, often in degenerative brain states. Delta waves include all the waves of the EEG with frequencies less than 3.5 cycles/sec. They occur in very deep sleep, in infancy, and in persons with serious organic brain disease [10].

In order to understand the mechanism of action of acupuncture practices especially in the central nervous system, it is important to determine the changes in brain waves, which occurred by the acupuncture application. There are many publications investigating the changes in EEG occurred by acupuncture application [11]-[16].

We, therefore, examined the changes in the EEG occurred by the application of acupuncture to the GV 20 point [11]. In this single-blind, randomized, cross-over study, we particularly chose the GV 20 point, which is used in the treatments of depression, anxiety, and panic attack and has a regulatory impact on psychological state. Verum Acupuncture (VA) was performed at GV 20 point in 17 volunteers and 1 week after, Placebo Acupuncture (PA) was performed at the sham point chosen on their scalp. EEG data were obtained before and after these applications. We compared the spectral analysis of EEG before and after the application. Accordingly, as compared the baseline data, an increase was observed in the alpha band power at the T4 and T5 electrodes ( $p$ $<0.05$ ) and a decrease was observed in the beta band power at the T4, T6, and A2 electrodes following the application of VA to GV 20 point. On the other hand, when the data recorded before and after PA were compared it was revealed that there were no significant differences between the data. The results of this study suggested that VA applied at GV 20 acupoint had an effect on brain activity and might have a regulatory impact on psychological state by relaxing the subjects (Table 1 ).

Table 1. Studies of EEG changes occurring due to acupuncture applications.

\begin{tabular}{|c|c|c|c|c|c|c|c|}
\hline & $\begin{array}{l}\text { Number of } \\
\text { Volunteers }\end{array}$ & Blindness & $\begin{array}{l}\text { Number of } \\
\text { Channels }\end{array}$ & $\begin{array}{l}\text { Stimulated } \\
\text { acupoints }\end{array}$ & $\begin{array}{l}\text { Stimulated } \\
\text { Sham Points }\end{array}$ & $\begin{array}{l}\text { Stimulation } \\
\text { Period }\end{array}$ & Results \\
\hline $\begin{array}{l}\text { Cabioğu } \\
\text { (2016) [11] }\end{array}$ & 17 & $\begin{array}{c}\text { Not } \\
\text { blinded }\end{array}$ & 21 & GV20 & Yes & Manual & Relaxation Effect \\
\hline $\begin{array}{l}\text { Rosted } \\
\text { (2001) [17] }\end{array}$ & 14 & Blinded & 1 & LI4 & Yes & Manual & No Significant Changes \\
\hline $\begin{array}{c}\text { Chen } \\
\text { (2006) [11] }\end{array}$ & 12 & Blinded & 124 & LI4 & Yes & EA & $\begin{array}{l}\text { Significant Change } \\
\text { with High Frequency }\end{array}$ \\
\hline $\begin{array}{l}\text { Litscher } \\
\text { (2006) [12] }\end{array}$ & 9 & $\begin{array}{l}\text { Partially } \\
\text { double } \\
\text { blinded }\end{array}$ & 124 & $\begin{array}{c}\text { GV20, Ex.1, Ex. } \\
\text { 9, He7, Pe6, } \\
\text { CV6, St36, Sp6 }\end{array}$ & None & $\begin{array}{c}\text { Manual } \\
\text { and laser }\end{array}$ & $\begin{array}{l}\text { Significant Changes } \\
\text { at Sedative Points }\end{array}$ \\
\hline $\begin{array}{l}\text { Sakai } \\
\text { (2007) [15] }\end{array}$ & 16 & $\begin{array}{c}\text { Not } \\
\text { blinded }\end{array}$ & 19 & $\begin{array}{l}\text { Ant fibers of } \\
\text { trapezius muscle }\end{array}$ & & & Significant Changes \\
\hline
\end{tabular}


In the study by Chang et al. [12], the effects of acupuncture at P6, which was chosen due to its association with the meridian concerning the circulatory system according to TCM, on the EEG were investigated. They included 12 male healthy volunteers having no neurological disease who were divided into 2 groups equally. They obtained data set of spectral analyses from 18 trials before, during, and after sham/manual acupuncture. While the needle was perpendicularly inserted into the P6 site in the manual acupuncture group, it was inserted in a slanting direction near the P6 site in the sham acupuncture group. EEG data at the baseline and after acupuncture were compared and the changes were obtained. In all trials, there was an increase in the amplitude and power of the alpha band during manual acupuncture. In the alpha band of 12-channel, the frequency peaks were synchronized. After the experiment, the high power and synchronized frequencies continued for at least 10 minutes in the manual acupuncture group. As a consequence, it was concluded that the high power and synchronized rhythms in the brain oscillations might be associated with the autonomic nervous system (Table 1).

Streitberger et al. [13] investigated the effects of acupuncture on central and autonomic nervous system and evaluated the changes in EEG and heart rate. They performed VA at the acupuncture point LI4 and PA at the sham point on 20 healthy volunteers with the mean age of $25.2 \pm 3.6$ years old. They also recorded the 12-channel EEG data. VA was performed by inserting stainless steel needle at the point LI 4 and PA was performed by inserting a blunted, telescopic placebo needle at the sham point. The subjects were randomly divided into two groups. In the first group, VA was performed at the point LI 4 at the first day and PA was performed at the sham point within a 2-week period. In the second group, PA was performed at first and then VA was performed. When comparing the applications of VA and PA, there was an increase in the alpha1 frequency of occipital region and alpha 1/teta was shifted to the benefit of alpha 1 for all electrodes $(p<0.04)$. No significant change was observed in the delta, beta, and alpha 2 frequencies. The ratio of low frequency/high frequency was significantly increased in the short term in the VA whereas, no such difference was observed for PA. In that study, it was concluded that changes in the parameters of heart rate variation and the increase in the ratio of low frequency/high frequency in the brain waves by VA application in the first minute reflected the enhancement of sympathetic activity at baseline. Observing an increase in the alpha 1 frequency in the occipital region by VA application particularly suggested that this was a specific effect of stimulation of the LI 4 point. That study strengthens the idea that acupuncture may affect the autonomic system (Table 1).

The study of Chen et al. [14] determined that effects of acupuncture were specific to EEG bands. 124-ch EEG data were used to analyze for brain wave. Twelve healthy male volunteers participated in two experimental sessions separated by 1 week, which involved transcutaneous acupoint stimulation at selected LI 4 acupoint vs. a mock point on the left hand in high (HF:100 Hz) vs. low-frequency (LF:2 Hz) stimulation. In this study the defined activity of the Theta power significantly decreased $(P=0.02)$, compared with control during HF but not LF stimulation at acupoint stimulation, however, there was no study effect at the mock point. A decreased Theta EEG power was featured at the frontal midline sites and the contralateral right hemisphere front site. The showed high-frequency acupoint stimulation effects of Theta EEG were only present during simulation. The topographic Theta activity was temporarily determined to originate from the intracranial current source in cingulate cortex. Chen and colleagues thought likely that short-term cortical plasticity occurred during high-frequency but not low-frequency stimulation at LI 4, but not sham point and suggested that LI 4 stimulation modulates limbic cingulum by a frequency modulation mode (Table 1).

Sakai et al. [15] was used acupuncture in therapy for chronic pain syndrome. The forebrain including the various cerebral cortices has been implicated in inhibitory and facilitatory control of pain as well as autonomic functions. Sakai et al. searched relationships among specific sensations induced by acupuncture manipulation, effects on sympathetic and parasympathetic autonomic functions, and EEG changes. An acupuncture needle was inserted into the right trapezius muscle of the subjects, and acupuncture manipulation was recurred to induce specific acupuncture sensation recurrently while the needle was left in the muscle. Acupuncture application pointedly decreased heart rate, and increased systolic blood pressure. Spectral analysis showed that acupuncture manipulation significantly decreased low frequency components of both heart rate variability and sistolic blood pressure variability, and significantly reduced ratio of low frequency to high frequency component (HF) of HRV. Index of sympathetic activity is LF/HF. These results are beneficial to relieve chronic pain by inhibiting sympathetic nervous activity (Table 1 ).

Litscher et al. [16] investigated response entropy (RE) and state entropy (SE) in acupuncture research. Both parameters have been alleged to reflect changes in the clinical state of sedation. Two different acupuncture plans were applied in a randomized crossover trial with nine healthy volunteers. The application of acupuncture 
needles or performing laser needle acupuncture at special sedation points decreased RE and SE significantly compared with the reference interval before acupuncture. Conversely, acupuncture of points for increasing "Qi-energy" did not decrease parameters of entropy. Therefore, entropy measurements during the application of acupuncture appear to be worthy of further evaluation that is to be studied in future period (Table 1).

The abovementioned studies have suggested that acupuncture practices applied to the different acupoints might lead to different brain activities in different regions of the brain by an increase in the alpha waves in the temporal region by the stimulation of acupoint GV 20, an increase in all regions by the stimulation of acupoint P6, and an increase in the alpha 1 waves in the occipital region by the stimulation of acupoint LI 4 might lead to different acupoints which can cause different brain activities in different regions of the brain. Besides, LI 4 stimulation as transcutaneous one modulates limbic cingulum by a frequency modulation mode. Moreover, an increase in the alpha wave frequency caused by the stimulation of acupoints in different meridian and in different regions has indicated that these points have mood modifying and relaxation effects and they can be used in the psychological state changes. And acupuncture with the analysis of EEG changes occurs; I think it will contribute to explaining the mechanism of effects of acupuncture.

\section{References}

[1] Moffet, H.H. (2006) How Might Acupuncture Work? A Systematic Review of Physiologic Rationales from Clinical Trials. BMC Complementary and Alternative Medicine, 6, 25. http://dx.doi.org/10.1186/1472-6882-6-25

[2] Zhao, Z.Q. (2008) Neural Mechanism Underlying Acupuncture Analgesia. Progress in Neurobiology, 85, 355-375. http://dx.doi.org/10.1016/j.pneurobio.2008.05.004

[3] Cabığlu, M.T. and Ergene, N. (2003) The Effect Mechanism of Acupuncture and Clinical Applications. Genel Tip Dergisi, 13, 35-40.

[4] Cabığlu, M.T. (2008) Pain Control with Acupuncture and Neurotransmitters. Genel Tip Dergisi, 18, 93-98.

[5] Cabioglu, M.T. and Surucu, S. (2009) Acupuncture and Neurophysiology. Medical Acupuncture, 21, 13-20. http://dx.doi.org/10.1089/acu.2009.0638

[6] Cabýoglu, M.T., Ergene, N. and Tan, U. (2006) The Mechanism of Acupuncture and Clinical Applications. International Journal of Neuroscience, 116, 115-125. http://dx.doi.org/10.1080/00207450500341472

[7] Cabioglu, M.T. and Arslan, G. (2008) Neurophysiologic Basis of Back-Shu and Huatuo-Jiaji Points. The American Journal of Chinese Medicine, 36, 473-479. http://dx.doi.org/10.1142/S0192415X08005916

[8] Cabioglu, M.T. and Surucu, S. (2009) Neurophysiologic Basis of Front-Mu Points. Neuroanatomy, 8, 32-35.

[9] Majumdar, K. (2011) Human Scalp EEG Processing: Various Soft Computing Approaches. Applied Soft Computing, 11, 4433-4447. http://dx.doi.org/10.1016/j.asoc.2011.07.004

[10] Guyton, A.C. and Hall, J.E. (2016) Textbook of Medical Physiology. WB Saunders, Philadelphia, 741-747.

[11] Chang, S., Chang, Z.G., Li, S.J., Chiang, M.J., Ma, C.M., Cheng, H.Y. and Hsieh, S.H. (2009) Effects of Acupuncture at Neiguan (PC 6) on Electroencephalogram. Chinese Journal of Physiology, 52, 1-7. http://dx.doi.org/10.4077/CJP.2009.AMG070

[12] Chen, A.C., Liu, F.J., Wang, L. and Arendt-Nielsen, L. (2006) Mode and Site of Acupuncture Modulation in the Human Brain: 3D (124-ch) EEG Power Spectrum Mapping and Source Imaging. Neuroimage, 29, 1080-1091. http://dx.doi.org/10.1016/j.neuroimage.2005.08.066

[13] Litscher, G. (2006) Electroencephalogram—Entropy and Acupuncture. Anesthesia \& Analgesia, 102, 1745-1751. http://dx.doi.org/10.1213/01.ane.0000217188.71710.47

[14] Cabioglu, M.T., KilincToprak, M., Y1lmaz, D. and Cabıglu, C. Effect of Acupuncture Stimulation of GV 20 Point on Electroencephalogram.

[15] Streitberger, K., Steppan, J., Maier, C., Hill, H., Backs, J. and Plaschke, K. (2008) Effects of Verum Acupuncture Compared to Placebo Acupuncture on Quantitative EEG and Heart Rate Variability in Healthy Volunteers. The Journal of Alternative and Complementary Medicine, 14, 505-513. http://dx.doi.org/10.1089/acm.2007.0552

[16] Chen, A.C., Liu, F.J., Wang, L. and Arendt-Nielsen, L. (2006) Mode and Site of Acupuncture Modulation in the Human Brain: 3D (124-ch) EEG Power Spectrum Mapping and Source Imaging. Neuroimage, 29, 1080-1091. http://dx.doi.org/10.1016/j.neuroimage.2005.08.066

[17] Sakai, S., Hori, E., Umeno, K., Kitabayashi, N., Ono, T. and Nishijo, H. (2007) Specific Acupuncture Sensation Correlates with EEGs and Autonomic Changes in Human Subjects. Autonomic Neuroscience: Basic and Clinical, 133, 158-169. http://dx.doi.org/10.1016/j.autneu.2007.01.001 